\title{
Detection of Linear Modulations in the Presence of Strong Phase and Frequency Instabilities
}

\author{
Giulio Colavolpe, Associate Member, IEEE, and Riccardo Raheli, Member, IEEE
}

\begin{abstract}
Noncoherent sequence detection algorithms, recently proposed by the authors, have a performance which approaches that of coherent detectors and are robust to phase and frequency instabilities. These schemes exhibit a negligible performance loss in the presence of a frequency offset, provided this offset does not exceed an order of $1 \%$ of the signaling frequency. For higher values, the performance rapidly degrades. In this paper, detection schemes are proposed, characterized by high robustness to frequency offsets and capable of tolerating offset values up to $10 \%$ of the signaling frequency. More generally, these detection schemes are very robust to rapidly varying phase and frequency instabilities. The general case of coded linear modulations is addressed, with explicit reference to $M$-ary phase shift keying and quadrature amplitude modulation.
\end{abstract}

Index Terms-Frequency estimation, maximum-likelihood detection, noncoherent sequence detection.

\section{INTRODUCTION}

$\mathbf{I}$ N BURST-MODE transmissions, typical of future-generation wireless local loops, mobile to satellite, and cellular mobile radio systems, a frequency offset is often present, possibly due to a Doppler shift or a mismatch in the frequency of transmit and receive up- and down-conversion oscillators. In this scenario, the use of efficient noncoherent detection or decoding schemes is often considered (see [1]-[5] and references therein). Noncoherent sequence detection (NSD) [1], [2] is a class of recently proposed schemes which allow us to approach the performance of optimal coherent receivers [1], [2], [6] for any coded linear and continuous-phase modulation format.

The starting point in [1] and [2] is the optimal noncoherent receiver which operates in the presence of a random phase rotation of the received signal, modeled as constant during the transmission, and additive white Gaussian noise (AWGN). Since optimal sequence detection requires a search of a path in a tree diagram, the required complexity increases exponentially with the duration of the transmission. In [1] and [2], suitable approximations are proposed in order to reduce the problem to a search of a path in a trellis diagram and realize simple suboptimal detection schemes based on a Viterbi algorithm. Besides being realizable, these suboptimal schemes have the convenient feature of allowing us to remove the constant phase assumption and encompassing time-varying phase models. In fact, NSD schemes are

Paper approved by L. Vandendorpe, the Editor for Transmission Systems of the IEEE Communications Society. Manuscript received July 15, 2000; revised June 15, 2001. This work was supported by Ministero dell'Università e della Ricerca Scientifica e Tecnologica (MURST), Italy. This paper was presented in part at the IEEE International Conference on Communications (ICC'00), New Orleans, LA, June 2000.

The authors are with the Dipartimento di Ingegneria dell'Informazione, Università di Parma, 43100 Parma, Italy.

Digital Object Identifier 10.1109/TCOMM.2002.804143 very robust to oscillator instabilities, such as phase noise, and do not require an acquisition period as in the case of coherent detection schemes based on a phase-locked loop (PLL). Compared to coherent schemes, they entail a negligible performance degradation as long as the uncompensated frequency offset is, at most, of the order of $1 \%$ of the signaling rate, whereas, for higher values, the performance rapidly degrades.

In this paper, we propose new detection algorithms characterized by high robustness to frequency offsets, practically limited by the suboptimality of the matched-filter front end only, at the price of a possible moderate degradation with respect to the performance exhibited by NSD schemes in the absence of frequency offsets. Although a matched-filter front end is conceptually suboptimal in the presence of a frequency offset, from a practical point of view, offsets up to $10 \%$ of the symbol rate can be tolerated.

We present detection schemes with high robustness to phase and frequency instabilities. The first scheme is based on feedforward frequency estimation and per-survivor processing (PSP) [7], embedded in an NSD algorithm. To this purpose, several frequency estimation algorithms are considered (see [8]-[11] and references therein). The other two detection strategies are totally invariant to frequency offsets and are based on ad hoc solutions. All detection strategies are introduced for general coded linear modulation formats. As they operate on the basis of short and undelayed observation windows, they tolerate rapidly varying phase and frequency instabilities. This is made possible by the use of PSP-based frequency estimation, which is explicit in the first proposed detection algorithm, and it is performed implicitly in the other proposed schemes.

In the next section, we review the assumed system model and the basic likelihood function. The proposed schemes are described in Sections III and IV. Specifically, in Section III, a detection algorithm which incorporates PSP-based frequency estimation is proposed. In Section IV, frequency-invariant detection algorithms are derived in the case of equal-energy signals and then extended to the case of nonequal-energy signals. In Section V, numerical results are presented. Finally, conclusions are drawn in Section VI.

\section{System Model AND LiKelihood FunCTION}

The assumed system model is shown in Fig. 1. The information sequence $\left\{a_{k}\right\}$, composed of independent and identically distributed (i.i.d.) symbols belonging to an $M$-ary alphabet, is mapped into a code sequence $\left\{c_{k}\right\}$ by means of some coding rule. This code sequence is further mapped by a linear modulator into a time-continuous signal with complex 


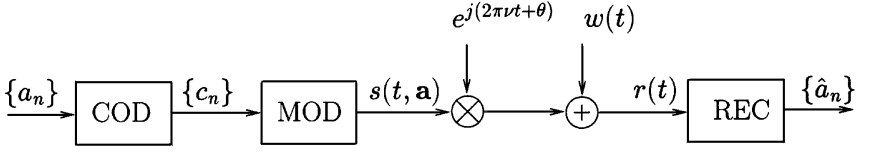

Fig. 1. System model.

envelope $s(t, \mathbf{a})$, which depends on the information sequence denoted by the vector $\mathbf{a}$. This signal undergoes a phase rotation $2 \pi \nu t+\theta$, and is affected by AWGN $w(t)$, which is the baseband equivalent of bandpass noise with two-sided power spectral density $N_{0} / 2$. The complex envelope of the received signal may be expressed as

$$
r(t)=s(t, \mathbf{a}) e^{j(2 \pi \nu t+\theta)}+w(t) .
$$

The phase $\theta$ is modeled as a random variable with uniform distribution in the interval $[0,2 \pi)$ and the frequency offset $\nu$ is assumed deterministic. Both parameters are initially assumed constant during the entire transmission, whereas this assumption will be relaxed later on. In the case of linearly modulated signals, the information-bearing signal may be expressed as

$$
s(t, \mathbf{a})=\sum_{k=0}^{N_{T}-1} c_{k} h(t-k T)
$$

where $N_{T}$ denotes the number of transmitted code symbols, $T$ is the signaling interval, and $h(t)$ is a properly normalized shaping pulse.

Assuming perfect symbol synchronization and absence of frequency offset, the output, sampled at time $t=k T$, of a filter matched to the shaping pulse $h(t)$ is a sufficient statistic for this detection problem. If a moderate frequency offset is present, this is not true in a strict sense; however, this sampled output may still be considered as an approximate sufficient statistic. This assumption is commonly used in the derivation of frequency estimation algorithms (see [10], [11] and references therein). ${ }^{1}$ With the further assumption of absence of intersymbol interference, i.e., $\left.h(t) \otimes h(-t)\right|_{t=k T}=\delta_{k}$ where $\otimes$ denote convolution and $\delta_{k}$ is the Kronecker delta, it is straightforward to show that the likelihood function for joint data detection and frequency estimation reads

$$
\begin{aligned}
\Gamma_{N_{T}}(\tilde{\mathbf{a}}, \tilde{\nu})=N_{0} \ln I_{0}\left(\frac{1}{N_{0}}\left|\sum_{k=0}^{N_{T}-1} x_{k} \tilde{c}_{k}^{*} e^{-j 2 \pi k \tilde{\nu} T}\right|\right) \\
-\frac{1}{2} \sum_{k=0}^{N_{T}-1}\left|\tilde{c}_{k}\right|^{2}
\end{aligned}
$$

where $\left\{\tilde{c}_{k}\right\}$ is the code sequence uniquely associated with a hypothetical information sequence $\tilde{\mathbf{a}}$ by the given coding rule, $\tilde{\nu}$ denotes a trial value of the frequency offset, and $x_{k} \triangleq r(t) \otimes$ $\left.h(-t)\right|_{t=k T}$ is the matched filter output, sampled at time $t=$ $k T$. In the following, samples $\left\{x_{k}\right\}$ will be referred to as "observations." The likelihood function (3) is similar to that obtained in [1] in the absence of frequency offsets. The likelihood function in [1] may be obtained by letting $\tilde{\nu}=0$ in (3).

A property of the likelihood function (3) which is of interest in this paper is now described. Let us assume that two code

\footnotetext{
${ }^{1}$ For larger values of the frequency offset $\nu$, a different front end, possibly based on oversampling techniques, could be used [12].
}

sequences, $\left\{c_{k}^{(1)}\right\}$ and $\left\{c_{k}^{(2)}\right\}$, corresponding to distinct information sequences, $\mathbf{a}^{(1)}$ and $\mathbf{a}^{(2)}$, respectively, exist such that $c_{k}^{(2)}=c_{k}^{(1)} e^{j\left(2 \pi \nu_{0} k T+\theta\right)}$, where $\nu_{0}$ is some frequency value and $\theta$ is some phase rotation. The structure of the likelihood function (3) implies that

$$
\Gamma_{N_{T}}\left(\mathbf{a}^{(1)}, \tilde{\nu}\right)=\Gamma_{N_{T}}\left(\mathbf{a}^{(2)}, \tilde{\nu}+\nu_{0}\right) .
$$

A consequence of this property is that if the coding rule admits such sequences, a decoding strategy based on (3), directly or by means of some approximations, will not be able to distinguish the information sequences $\mathbf{a}^{(1)}$ and $\mathbf{a}^{(2)}$.

For generality, the concept of phase rotation of order $l$ may be introduced. A time-varying phase rotation $\phi_{k}$ is said to be of order $l$ if it may be expressed as

$$
\phi_{k}=\phi^{(l)}(k T)^{l}+\phi^{(l-1)}(k T)^{l-1}+\cdots+\phi^{(1)} k T+\phi^{(0)}
$$

where $\phi^{(l)}, \ldots, \phi^{(0)}$ are suitable constants. The above property (4) may be reformulated in the following terms: two code sequences which differ for a phase rotation of the first order are indistinguishable. For this reason, a code which admits code sequences that differ for a first-order phase shift is catastrophic when decoded by means of strategy (3). Note that this problem resembles, in a higher-order sense, the usual characteristic of noncoherent receivers of being unable to distinguish code sequences which differ for a phase rotation of zeroth order [1]. Although the usual differential encoding (DE) rule solves this zeroth-order ambiguity, it is catastrophic in a first-order sense. In fact, DE has the property that two code sequences which differ for a zeroth-order phase rotation are associated with the same information sequence, but code sequences which differ for a first-order phase rotation may derive from different information sequences.

These concepts can be generalized to phase rotations of second or higher order. An example of a second-order phase rotation is of interest in low-earth-orbit satellite systems affected by time-varying Doppler shifts [13].

\section{PSP-BASED DETECTION AlgORITHM}

In order to perform data detection, the method of generalized likelihood [14], i.e., the joint maximization of (3) with respect to $\tilde{\mathbf{a}}$ and $\tilde{\nu}$, is equivalent to the maximization of the following likelihood function:

$$
\begin{aligned}
\Lambda_{N_{T}}(\tilde{\mathbf{a}}) \triangleq & \Gamma_{N_{T}}(\tilde{\mathbf{a}}, \hat{\nu}(\tilde{\mathbf{a}})) \\
= & N_{0} \ln I_{0}\left(\frac{1}{N_{0}}\left|\sum_{k=0}^{N_{T}-1} x_{k} \tilde{c}_{k}^{*} e^{-j 2 \pi k \hat{\nu}(\tilde{\mathbf{a}}) T}\right|\right) \\
& -\frac{1}{2} \sum_{k=0}^{N_{T}-1}\left|\tilde{c}_{k}\right|^{2}
\end{aligned}
$$

where

$$
\hat{\nu}(\tilde{\mathbf{a}}) \triangleq \arg \max _{\tilde{\nu}}\left|\sum_{k=0}^{N_{T}-1} x_{k} \tilde{c}_{k}^{*} e^{-j 2 \pi k \tilde{\nu} T}\right|
$$

may be interpreted as a PSP-based maximum-likelihood frequency estimate [7]. 
Some approximations are now introduced in order to realize simple suboptimal detection schemes based on a Viterbi algorithm. In (6), we use the standard approximation $\ln \left[I_{0}(x)\right] \simeq x$, valid for large values of the argument [1], [4]. On the correct path, the longer the transmission length $N_{T}$, the better the approximation quality for a given signal-to-noise ratio. Proceeding as in [1], we may define the following partial sequence and incremental metrics

$$
\begin{aligned}
\Lambda_{n}\left(\tilde{\mathbf{a}}_{n-1}\right) & \triangleq\left|\sum_{k=0}^{n-1} x_{k} \tilde{c}_{k}^{*} e^{-j 2 \pi k \hat{\nu}\left(\tilde{\mathbf{a}}_{n-1}\right) T}\right|-\frac{1}{2} \sum_{k=0}^{n-1}\left|\tilde{c}_{k}\right|^{2} \\
\Delta_{n}\left(\tilde{\mathbf{a}}_{n}\right) \triangleq & \Lambda_{n+1}\left(\tilde{\mathbf{a}}_{n}\right)-\Lambda_{n}\left(\tilde{\mathbf{a}}_{n-1}\right) \\
= & \left|\sum_{k=0}^{n} x_{k} \tilde{c}_{k}^{*} e^{-j 2 \pi k \hat{\nu}\left(\tilde{\mathbf{a}}_{n}\right) T}\right| \\
& -\left|\sum_{k=0}^{n-1} x_{k} \tilde{c}_{k}^{*} e^{-j 2 \pi k \hat{\nu}\left(\tilde{\mathbf{a}}_{n-1}\right) T}\right|-\frac{1}{2}\left|\tilde{c}_{n}\right|^{2}
\end{aligned}
$$

where $\tilde{\mathbf{a}}_{n}$ is the hypothetical information sequence up to discrete time $n$. As in [1], in order to limit the memory of the incremental metric (9), a truncation is introduced to enable a search of a trellis diagram by means of a Viterbi algorithm. To this purpose, in (9) we may consider $N \ll N_{T}$ most recent observations $x_{k}$ and code symbols $\tilde{c}_{k}$. In addition, we substitute the two frequency estimates $\hat{\nu}\left(\tilde{\mathbf{a}}_{n}\right)$ and $\hat{\nu}\left(\tilde{\mathbf{a}}_{n-1}\right)$, with a single estimate $\hat{\nu}_{L}\left(\tilde{\mathbf{a}}_{n}\right)$ based on the $L \ll N_{T}$ most recent observations $x_{k}$ and code symbols $\tilde{c}_{k}$. In analogy with [1], integers $N$ and $L$ represent the assumed phase and frequency memory, respectively. After an initial transient period, i.e., for $n \geq \max \{N, L\}-1$, the resulting branch metrics are ${ }^{2}$

$$
\begin{aligned}
\lambda_{n}\left(\tilde{\mathbf{a}}_{n}\right)= & \left|\sum_{i=0}^{N-1} x_{n-i} \tilde{c}_{n-i}^{*} e^{-j 2 \pi(n-i) \hat{\nu}_{L}\left(\tilde{\mathbf{a}}_{n}\right) T}\right| \\
& -\left|\sum_{i=1}^{N-1} x_{n-i} \tilde{c}_{n-i}^{*} e^{-j 2 \pi(n-i) \hat{\nu}_{L}\left(\tilde{\mathbf{a}}_{n}\right) T}\right|-\frac{1}{2}\left|\tilde{c}_{n}\right|^{2} .
\end{aligned}
$$

Thanks to the introduced memory truncation, the initial assumption of constant phase and frequency offset during the entire transmission may be significantly relaxed. In fact, these channel parameters must be approximately constant in an interval of $\max (L, N)$ symbols, only. As shown in the numerical results, practical values of $N$ and $L$ are rather small; hence, these channel parameters are allowed to change rapidly with time.

The frequency estimate $\hat{\nu}_{L}\left(\tilde{\mathbf{a}}_{n}\right)$ may be obtained from maximum-likelihood data-aided estimation based on the algorithm by Rife and Boorstyn [8]. However, in general, a simpler dataaided algorithm may be used. We consider some of the algorithms described in [11], namely those by Kay [9] and Mengali and Morelli [10]. In the technical literature, these algorithms have been used for $M$-ary phase shift keying ( $M$-PSK) signals [10], [11]. However, they may be easily extended to the general case of nonequal-energy signals such as quadrature amplitude modulation ( $M$-QAM), as shown in the Appendix.

Memory truncation provides significant benefits in channels with high dynamics, at the price of a reduced estimation accu-
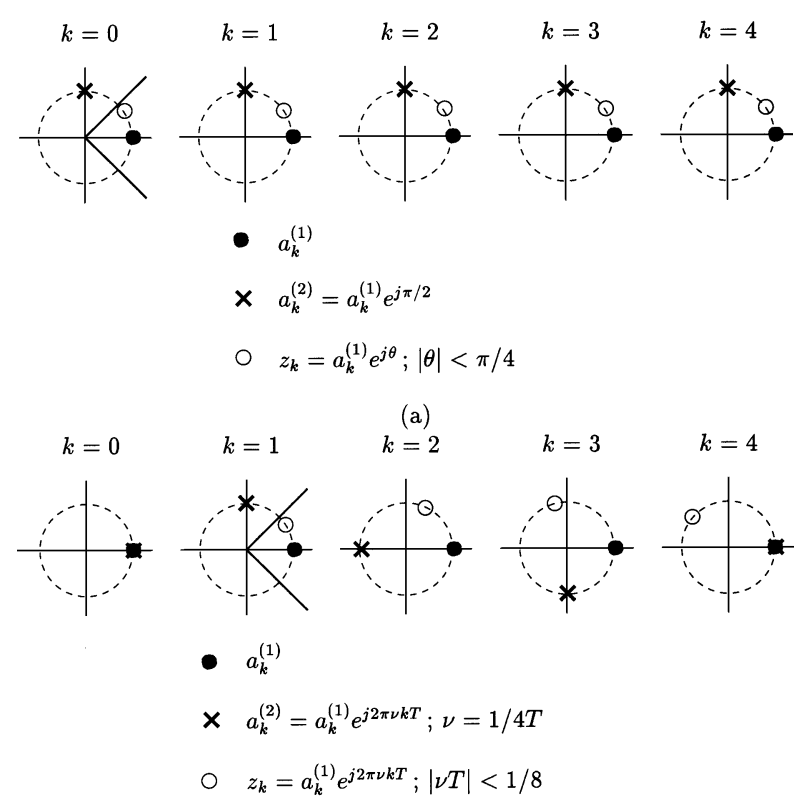

(b)

Fig. 2. Examples of indistinguishable sequence. (a) Noncoherent receiver (zeroth order). (b) Proposed receiver (first order).

racy in channels with low dynamics. A quantitative evaluation of the relevant degradation is performed in [8]-[11] for various frequency estimation algorithms, in terms of variance of the estimation error for a static channel. When these algorithms are used in conjunction with a noncoherent detection scheme, which is extremely robust to phase jitter and frequency instabilities [1], [2], a proper performance measure is the overall bit-error rate (BER) penalty with respect to an ideal coherent receiver (which perfectly knows the actual value of phase and frequency). In fact, these noncoherent schemes may exhibit good performance even with a rough estimation accuracy. In the numerical results, it is shown that for rather small values of $N$ and $L$, the performance penalty is limited and a high robustness is achieved.

The proposed detector with branch metrics (10) may be affected by the catastrophic property (4). In order to overcome this problem, we propose two possible solutions which can be derived by extending simple countermeasures for the zeroth-order ambiguity problems of noncoherent receivers to the first-order case. An example of indistinguishable sequences in a noncoherent receiver for uncoded quaternary PSK (QPSK) modulation is shown in Fig. 2(a). Sequence $\mathbf{a}^{(2)}$ is a rotation of sequence $\mathbf{a}^{(1)}$ by $\pi / 2$ and cannot be distinguished. However, if the phase rotation $\theta$ is limited as $|\theta|<\pi / 4$, as in the case of sequence $\left\{z_{k}\right\}$, the sequence $\mathbf{a}^{(1)}$ can be correctly recovered. Alternatively, differential encoding/decoding associates code sequences which differ for a constant phase shift with a same information sequence, eliminating this ambiguity. In Fig. 2(b), a first-order analogy is shown. Sequence $\mathbf{a}^{(2)}$ is a first-order rotation of sequence $\mathbf{a}^{(1)}$ with $\nu T=1 / 4$ and cannot be distinguished. ${ }^{3}$ However, if the frequency offset is limited as $|\nu T|<$ $1 / 8$, as in the case of sequence $\left\{z_{k}\right\}$, the ambiguity may be resolved. Alternatively, double differential encoding (DDE) with

${ }^{3} \operatorname{In}(5), \phi^{(1)}=2 \pi \nu$.

${ }^{2}$ For $n<\max \{N, L\}-1$, the two summations in (10) and/or the frequency estimate should be computed on a lower number of terms. 
suitable differential decoding may be adopted [5], [15]. These solutions are described in the following.

The first solution (method 1) consists of a limitation of the frequency offset range and estimation interval. Let us denote by $\psi_{0}$ the angle of invariance of the considered constellation. In the case of $M$-PSK signals, $\psi_{0}=2 \pi / M$, whereas, in the case of square $M$-QAM, $\psi_{0}=\pi / 2$. Being $2 \pi \nu T$ the phase rotation introduced by the channel in one signaling interval, when $-\psi_{0} / 2 \leq 2 \pi \nu T \leq \psi_{0} / 2$, this phase rotation does not yield ambiguities. At the same time, this condition should be also satisfied by the estimate $\hat{\nu}_{L}\left(\tilde{\mathbf{a}}_{n}\right)$. To this purpose, each estimate such that $\left|2 \pi \hat{\nu}_{L}\left(\tilde{\mathbf{a}}_{n}\right) T\right|>\psi_{0} / 2$ may be replaced by the closest value in the allowed interval. This method may be applied for limited values of the frequency offset only. In fact, for QPSK, the condition $|\nu T| \leq 1 / 8=0.125$ must be satisfied, whereas the allowed interval reduces to $|\nu T| \leq 0.03125$ for 16-PSK constellations. To avoid zeroth-order ambiguities, DE can be used.

An alternative solution (method 2) consists in the use of DDE, possibly followed by a code invariant to first-order rotations. ${ }^{4}$ As a consequence, unlike the previous case in which a limitation of the estimation interval must be imposed at the receiver, this solution does not require any modifications of the receiver operation (except for the fact that the new encoding rule must be taken into account). DDE is described for $M$-PSK modulations in Chapter 8 of [5] and [15]. In this case, symbols $\left\{c_{k}\right\}$ belonging to an $M$-PSK alphabet are derived from symbols $\left\{a_{k}\right\}$, belonging to the same alphabet, by means of the DDE rule

$$
c_{k}=a_{k} c_{k-1}^{2} c_{k-2}^{*} .
$$

In the case of $M$-QAM signals, quadrant [1] DDE may be used. Expressing the generic information symbol of an $M$-QAM constellation as $a_{k}=\mu_{k} p_{k}$, where $\mu_{k}$ belongs to the first quadrant and $p_{k} \in\{ \pm 1, \pm j\}$, the encoded symbol $c_{k}$ is given by $c_{k}=\mu_{k} q_{k}$, where $q_{k} \in\{ \pm 1, \pm j\}$ are defined by the DDE rule for QPSK modulations applied to symbols $\left\{p_{k}\right\}$, i.e., according to the rule

$$
q_{k}=p_{k} q_{k-1}^{2} q_{k-2}^{*}
$$

\section{FREQUENCY-INVARIANT DETECTION ALGORITHMS}

In this section, some detection schemes invariant with respect to the frequency offset are described. We explicitly consider the cases of equal-energy signals, such as $M$-PSK, and nonequalenergy signals, such as $M$-QAM.

\section{A. M-PSK Signals}

In the case of equal-energy signals, discarding irrelevant terms, the joint likelihood function (3) becomes

$$
\begin{aligned}
\Gamma_{N_{T}}(\tilde{\mathbf{a}}, \tilde{\nu})= & \left|\sum_{k=0}^{N_{T}-1} x_{k} \tilde{c}_{k}^{*} e^{-j 2 \pi k \tilde{\nu} T}\right| \sim\left|\sum_{k=0}^{N_{T}-1} x_{k} \tilde{c}_{k}^{*} e^{-j 2 \pi k \tilde{\nu} T}\right|^{4} \\
= & \sum_{n=0}^{N_{T}-1} \sum_{k=0}^{N_{T}-1} \sum_{i=0}^{N_{T}-1} \sum_{j=0}^{N_{T}-1} x_{n} \tilde{c}_{n}^{*} x_{k} \tilde{c}_{k}^{*} x_{i}^{*} \tilde{c}_{i} x_{j}^{*} \tilde{c}_{j} \\
& \cdot e^{-j 2 \pi(n+k-i-j) \tilde{\nu} T}
\end{aligned}
$$

${ }^{4}$ These codes may be viewed as an extension of the usual rotationally invariant codes [16] and are beyond the scope of this paper. where the expression $x \sim y$ denotes that $x$ and $y$ are monotonically related quantities. In this sum, each term such that $n+k-i-j=0$ is independent of $\nu$. Retaining only these terms, we obtain a simplified likelihood function which is invariant with respect to the parameter $\nu$. Observing that for each term in this quadruple summation there exists a complex conjugate term, and discarding terms independent of the code sequence $\left\{\tilde{c}_{k}\right\}$, we obtain the following equivalent simplified likelihood function:

$$
\begin{aligned}
\Lambda_{N_{T}}(\tilde{\mathbf{a}}) \triangleq \operatorname{Re}\left\{\sum_{n=2}^{N_{T}-1} x_{n} \tilde{c}_{n}^{*} \sum_{m=1}^{n-1} x_{n-m}^{*} \tilde{c}_{n-m}\right. & \\
& \left.\cdot \sum_{l=1}^{n-m} x_{n-m-l} x_{n-l}^{*} \tilde{c}_{n-m-l}^{*} \tilde{c}_{n-l}\right\}
\end{aligned}
$$

which may be recursively computed using the following incremental metric:

$$
\begin{aligned}
\Delta(\tilde{\mathbf{a}}) \triangleq \operatorname{Re}\left\{x_{n} \tilde{c}_{n}^{*} \sum_{m=1}^{n-1} x_{n-m}^{*} \tilde{c}_{n-m}\right. & \\
& \left.\cdot \sum_{l=1}^{n-m} x_{n-m-l} x_{n-l}^{*} \tilde{c}_{n-m-l}^{*} \tilde{c}_{n-l}\right\} .
\end{aligned}
$$

A truncated-memory branch metric may be defined by a procedure similar to that used to derive (10). For generality, we introduce two parameters, $N$ and $L$, in order to perform a different truncation in the two summations in (15). As an example, for $L \geq N$, we obtain

$$
\begin{aligned}
\lambda_{n}(\tilde{\mathbf{a}})=\operatorname{Re}\left\{x_{n} \tilde{c}_{n}^{*} \sum_{m=1}^{N-2} x_{n-m}^{*} \tilde{c}_{n-m}\right. \\
\left.\quad \cdot \sum_{l=1}^{L-m-1} x_{n-m-l} x_{n-l}^{*} \tilde{c}_{n-m-l}^{*} \tilde{c}_{n-l}\right\} .
\end{aligned}
$$

Note that raising the original likelihood function to the fourth power yields several terms. Some of these terms are then discarded. The effect of this approximation is a priori unknown and may be evaluated only by numerically simulating the performance of the derived suboptimal detection algorithm.

An interpretation of this branch metric is the following. Under the assumption of sufficiently small values of $\nu$, a coherent receiver for coded $M$-PSK selects the sequence $\left\{\tilde{c}_{n}\right\}$ which maximizes the sum of the branch metrics $\operatorname{Re}\left\{x_{n} \tilde{c}_{n} e^{-j(2 \pi n \nu T+\theta)}\right\}$, in which $\theta$ and $\nu$ are the correct channel phase and frequency offset. Extending the interpretations in [1], in (16) the inner sum (over index $l$ ) and the double summation may be interpreted as implicit PSP-based estimates of the phasors $e^{-j 2 \pi m \nu T}$ and $e^{-j(2 \pi n \nu T+\theta)}$, respectively, except for a normalization factor. Based on this remark, $N$ and $L$ can be interpreted as phase and frequency memory parameters, in analogy with the previous algorithm described in Section III. Similarly to the previous algorithm, in order to avoid a catastrophic behavior, the implicit frequency estimate must be limited within a suitable interval or DDE must be employed. We verified by computer simulation that the condition $L \geq N$ for the validity of (16) is of interest in practice, because the implicit estimation of the frequency offset is typically more critical than that of the phase. 
A less complex detection algorithm may be obtained by a different heuristic manipulation of the joint likelihood function (3). This likelihood function may be equivalently expressed as

$$
\begin{aligned}
\Gamma_{N_{T}}(\tilde{\mathbf{a}}, \tilde{\nu}) & \left|\sum_{k=0}^{N_{T}-1} x_{k} \tilde{c}_{k}^{*} e^{-j 2 \pi k \tilde{\nu} T}\right| \sim\left|\sum_{k=0}^{N_{T}-1} x_{k} \tilde{c}_{k}^{*} e^{-j 2 \pi k \tilde{\nu} T}\right|^{2} \\
= & \sum_{k=0}^{N_{T}-1} \sum_{m=0}^{N_{T}-1} x_{k} x_{m}^{*} \tilde{c}_{k}^{*} \tilde{c}_{m} e^{-j 2 \pi(k-m) \tilde{\nu} T} \\
= & \sum_{k=0}^{N_{T}-1}\left|x_{k}\right|^{2}\left|\tilde{c}_{k}\right|^{2} \\
& +2 \operatorname{Re}\left\{\sum_{k=1}^{N_{T}-1} x_{k} \tilde{c}_{k}^{*} \sum_{m=0}^{k-1} x_{m}^{*} \tilde{c}_{m} e^{-j 2 \pi(k-m) \tilde{\nu} T}\right\}
\end{aligned}
$$

in which the metric has been expressed as a sum of all the elements of a $N_{T} \times N_{T}$ Hermitian matrix with elements $x_{k} x_{m}^{*} \tilde{c}_{k}^{*} \tilde{c}_{m} e^{-j 2 \pi(k-m) \tilde{\nu} T}$. The first sum in (17) is independent of the code sequence because $\left|\tilde{c}_{k}\right|^{2}=1$. Thus, an equivalent sequence metric is

$$
\begin{aligned}
\Gamma_{N_{T}}(\tilde{\mathbf{a}}, \tilde{\nu}) & =\operatorname{Re}\left\{\sum_{k=1}^{N_{T}-1} x_{k} \tilde{c}_{k}^{*} \sum_{m=0}^{k-1} x_{m}^{*} \tilde{c}_{m} e^{-j 2 \pi(k-m) \tilde{\nu} T}\right\} \\
= & \operatorname{Re}\left\{\sum_{k=1}^{N_{T}-1} x_{k} \tilde{c}_{k}^{*} \sum_{i=1}^{k} x_{k-i}^{*} \tilde{c}_{k-i} e^{-j 2 \pi i \tilde{\nu} T}\right\} \\
= & \sum_{i=1}^{N_{T}-1} \operatorname{Re}\left\{\sum_{k=i}^{N_{T}-1} x_{k} \tilde{c}_{k}^{*} x_{k-i}^{*} \tilde{c}_{k-i} e^{-j 2 \pi i \tilde{\nu} T}\right\} \\
= & \sum_{i=1}^{N_{T}-1}\left|\sum_{k=i}^{N_{T}-1} x_{k} \tilde{c}_{k}^{*} x_{k-i}^{*} \tilde{c}_{k-i}\right| \cos \left\{\alpha_{i}(\mathbf{x}, \tilde{\mathbf{c}})-i \tilde{\nu} T\right\}
\end{aligned}
$$

in which

$$
\alpha_{i}(\mathbf{x}, \tilde{\mathbf{c}}) \triangleq \arg \left\{\sum_{k=i}^{N_{T}-1} x_{k} \tilde{c}_{k}^{*} x_{k-i}^{*} \tilde{c}_{k-i}\right\} .
$$

It is easy to verify that, in the absence of noise and for $\tilde{\mathbf{c}}=$ c, i.e., considering the transmitted code sequence, the generic term $\alpha_{i}(\mathbf{x}, \mathbf{c})$ is equal to $i \nu T .{ }^{5}$ Hence, for $\tilde{\nu}=\nu$ all the cosine factors in (18) are unitary. This fact suggests that the following approximate metric, asymptotically exact when the signal-tonoise ratio is high, can be used:

$$
\Lambda_{N_{T}}(\tilde{\mathbf{a}}) \triangleq \sum_{i=1}^{N_{T}-1}\left|\sum_{k=i}^{N_{T}-1} x_{k} \tilde{c}_{k}^{*} x_{k-i}^{*} \tilde{c}_{k-i}\right| .
$$

Proceeding as in Section III, we may define a partial sequence metric as

$$
\Lambda_{n}\left(\tilde{\mathbf{a}}_{n-1}\right) \triangleq \sum_{i=1}^{n-1}\left|\sum_{k=i}^{n-1} x_{k} \tilde{c}_{k}^{*} x_{k-i}^{*} \tilde{c}_{k-i}\right|
$$

and an incremental metric $\Delta_{n}\left(\tilde{\mathbf{a}}_{n}\right) \triangleq \Lambda_{n+1}\left(\tilde{\mathbf{a}}_{n}\right)-\Lambda_{n}\left(\tilde{\mathbf{a}}_{n-1}\right)$. The corresponding truncated-memory branch metric is

$$
\begin{aligned}
\lambda_{n}(\tilde{\mathbf{a}})=\sum_{i=1}^{N-2} & {\left[\left|\sum_{m=0}^{L-i-1} x_{n-m} \tilde{c}_{n-m}^{*} x_{n-m-i}^{*} \tilde{c}_{n-m-i}\right|\right.} \\
& \left.-\left|\sum_{m=1}^{L-i-1} x_{n-m} \tilde{c}_{n-m}^{*} x_{n-m-i}^{*} \tilde{c}_{n-m-i}\right|\right]
\end{aligned}
$$

where the parameters $N$ and $L$ have been introduced.

\section{B. M-QAM Signals}

In the case of nonequal-energy signals, it is not possible to directly apply the approximations of metric (3) described in the previous section. For this reason, as a starting point we use a noncoherent strategy recently derived under the assumption of Rayleigh fading and high signal-to-noise ratio, which, however, exhibits a very good performance in the case of Rice fading and AWGN channels as well [17]. The relevant likelihood function, extended to the case of an unknown frequency offset as described in Section II, is shown in (23) at the bottom of the page, where manipulations similar to those previously used in the case of equal-energy signals have been performed. As in the previous section, we consider the terms of the numerator with $n+k-i-j=0$, which are independent of the frequency offset $\nu$, and the corresponding terms in the denominator for proper normalization. The resulting simplified likelihood function may be expressed as shown in (24) at the bottom of the next page. Proceeding as in Section III, we may define

${ }^{5}$ Therefore, $\alpha_{i}(\mathbf{x}, \tilde{\mathbf{c}}) / i T$ may be interpreted as an estimate of $\nu$ based on PSP [7].

$$
\begin{aligned}
\Gamma_{N_{T}}(\tilde{\mathbf{a}}, \tilde{\nu}) & =\frac{\left|\sum_{k=0}^{N_{T}-1} x_{k} \tilde{c}_{k}^{*} e^{-j 2 \pi k \tilde{\nu} T}\right|^{2}}{\sum_{k=0}^{N_{T}-1}\left|\tilde{c}_{k}\right|^{2}} \sim \frac{\left|\sum_{k=0}^{N_{T}-1} x_{k} \tilde{c}_{k}^{*} e^{-j 2 \pi k \tilde{\nu} T}\right|^{4}}{\left[\sum_{k=0}^{N_{T}-1}\left|\tilde{c}_{k}\right|^{2}\right]^{2}} \\
& =\frac{\left|\sum_{k=0}^{N_{T}-1} x_{k} \tilde{c}_{k}^{*} e^{-j 2 \pi k \tilde{\nu} T}\right|^{4}}{\sqrt{\left[\sum_{k=0}^{N_{T}-1}\left|\tilde{c}_{k}\right|^{2}\right]^{4}}} \\
& =\frac{\sum_{n=0}^{N_{T}-1} \sum_{k=0}^{N_{T}-1} \sum_{i=0}^{N_{T}-1} \sum_{j=0}^{N_{T}-1} x_{n} \tilde{c}_{n}^{*} x_{k} \tilde{c}_{k}^{*} x_{i}^{*} \tilde{c}_{i} x_{j}^{*} \tilde{c}_{j} e^{-j 2 \pi(n+k-i-j) \tilde{\nu} T}}{\sqrt{\sum_{n=0}^{N_{T}-1} \sum_{k=0}^{N_{T}-1} \sum_{i=0}^{N_{T}-1} \sum_{j=0}^{N_{T}-1}\left|\tilde{c}_{n}\right|^{2}\left|\tilde{c}_{k}\right|^{2}\left|\tilde{c}_{i}\right|^{2}\left|\tilde{c}_{j}\right|^{2}}}
\end{aligned}
$$


a partial sequence metric and an incremental metric $\Delta_{n}\left(\tilde{\mathbf{a}}_{n}\right) \triangleq$ $\Lambda_{n+1}\left(\tilde{\mathbf{a}}_{n}\right)-\Lambda_{n}\left(\tilde{\mathbf{a}}_{n-1}\right)$. The corresponding truncated-memory branch metric is shown in (25) at the bottom of the page, for $L \geq N$.

The second heuristic approach, used in the previous section, may also be used for nonequal-energy signals, leading to a less complex detection algorithm with only slightly worse performance. The joint likelihood function (23) may be expressed as shown in (26) at the bottom of the page. Proceeding as in Section IV-A by assuming all the cosine factors in (26) unitary, we may choose to maximize the sequence metric shown in (27) at the bottom of the page. Defining a partial sequence metric and an incremental metric, the corresponding branch metric becomes as shown in (28) at the bottom of the page.

\section{NUMERICAL RESULTS}

The performance of the proposed detection algorithms is assessed by means of computer simulation in terms of BER versus $E_{b} / N_{0}, E_{b}$ being the average received signal energy per information bit.
As in [1], the state complexity of the proposed detection schemes may be limited by reduced-state sequence detection (RSSD) [18], [19]. This technique allows us to choose independently the parameters $N$ and $L$ from the number of states $S$ of the Viterbi algorithm. Hence, the number of states may be limited, retaining sufficiently large values for $N$ and $L$. In order to compute the branch metrics (10), (16), (22), (25), or (28) in a reduced trellis, the necessary symbols not included or not completely specified in the state definition are found in the survivor history according to PSP [7].

The performance of the receiver based on branch metrics (10) for QPSK with differential encoding (DQPSK), $N=7$, $S=16$, various values of $L$, and limitation of the estimation interval (method 1), is shown in Fig. 3. The algorithm by Kay [9] has been used for frequency estimation, but no differences were observed using more complex algorithms such as those in [8] and [10]. The optimal coherent receiver and an NSD receiver with $N=7$ and $S=16$ are also considered for comparison. The frequency offset is $\nu=0$. It may be observed that, for increasing values of $L$, the performance of the NSD receiver

$$
\Lambda_{N_{T}}(\tilde{\mathbf{a}}) \triangleq \frac{\operatorname{Re}\left\{\sum_{k=1}^{N_{T}-1} x_{k} \tilde{c}_{k}^{*} \sum_{m=0}^{k-1} x_{k-m}^{*} \tilde{c}_{k-m} \sum_{l=1}^{k-m} x_{k-l}^{*} \tilde{c}_{k-l} x_{k-m-l} \tilde{c}_{k-m-l}^{*}\right\}+\frac{1}{4} \sum_{k=0}^{N_{T}-1}\left|x_{k}\right|^{4}\left|\tilde{c}_{k}\right|^{4}}{\sqrt{\sum_{k=1}^{N_{T}-1}\left|\tilde{c}_{k}\right|^{2} \sum_{m=0}^{k-1}\left|\tilde{c}_{k-m}\right|^{2} \sum_{l=1}^{k-m}\left|\tilde{c}_{k-l}\right|^{2}\left|\tilde{c}_{k-m-l}\right|^{2}+\frac{1}{4} \sum_{k=0}^{N_{T}-1}\left|\tilde{c}_{k}\right|^{8}}}
$$

$$
\begin{aligned}
\lambda_{n}(\tilde{\mathbf{a}}) & =\frac{\operatorname{Re}\left\{\sum_{k=n-N+1}^{n} x_{k} \tilde{c}_{k}^{*} \sum_{m=0}^{k-n+N-2} x_{k-m}^{*} \tilde{c}_{k-m} \sum_{l=1}^{k-n-m+L-1} x_{k-l}^{*} \tilde{c}_{k-l} x_{k-m-l} \tilde{c}_{k-m-l}^{*}\right\}+\frac{1}{4} \sum_{k=n-N+1}^{n}\left|x_{k}\right|^{4}\left|\tilde{c}_{k}\right|^{4}}{\sqrt{\sum_{k=n-N+1}^{n}\left|\tilde{c}_{k}\right|^{2} \sum_{m=0}^{k-n+N-2}\left|\tilde{c}_{k-m}\right|^{2} \sum_{l=1}^{k-n-m+L-1}\left|\tilde{c}_{k-l}\right|^{2}\left|\tilde{c}_{k-m-l}\right|^{2}+\frac{1}{4} \sum_{k=n-N+1}^{n}\left|\tilde{c}_{k}\right|^{8}}} \\
- & \frac{\operatorname{Re}\left\{\sum_{k=n-N+1}^{n-1} x_{k} \tilde{c}_{k}^{*} \sum_{m=0}^{k-n+N-2} x_{k-m}^{*} \tilde{c}_{k-m} \sum_{l=1}^{k-n-m+L-1} x_{k-l}^{*} \tilde{c}_{k-l} x_{k-m-l} \tilde{c}_{k-m-l}^{*}\right\}+\frac{1}{4} \sum_{k=n-N+1}^{n-1}\left|x_{k}\right|^{4}\left|\tilde{c}_{k}\right|^{4}}{\sqrt{\sum_{k=n-N+1}^{n-1}\left|\tilde{c}_{k}\right|^{2} \sum_{m=0}^{k-n+N-2}\left|\tilde{c}_{k-m}\right|^{2} \sum_{l=1}^{k-n-m+L-1}\left|\tilde{c}_{k-l}\right|^{2}\left|\tilde{c}_{k-m-l}\right|^{2}+\frac{1}{4} \sum_{k=n-N+1}^{n-1}\left|\tilde{c}_{k}\right|^{8}}}
\end{aligned}
$$

$$
\begin{aligned}
\Gamma_{N_{T}}(\tilde{\mathbf{a}}, \tilde{\nu}) & =\frac{\left|\sum_{k=0}^{N_{T}-1} x_{k} \tilde{c}_{k}^{*} e^{-j 2 \pi k \tilde{\nu} T}\right|^{2}}{\sum_{k=0}^{N_{T}-1}\left|\tilde{c}_{k}\right|^{2}} \\
& =\frac{\frac{1}{2} \sum_{k=0}^{N_{T}-1}\left|x_{k}\right|^{2}\left|\tilde{c}_{k}\right|^{2}+\sum_{i=1}^{N_{T}-1} \operatorname{Re}\left\{\sum_{k=i}^{N_{T}-1} x_{k} \tilde{c}_{k}^{*} x_{k-i}^{*} \tilde{c}_{k-i} e^{-j 2 \pi i \tilde{\nu} T}\right\}}{\frac{1}{2} \sum_{k=0}^{N_{T}-1}\left|\tilde{c}_{k}\right|^{2}} \\
& =\frac{\frac{1}{2} \sum_{k=0}^{N_{T}-1}\left|x_{k}\right|^{2}\left|\tilde{c}_{k}\right|^{2}+\sum_{i=1}^{N_{T}-1}\left|\sum_{k=i}^{N_{T}-1} x_{k} \tilde{c}_{k}^{*} x_{k-i}^{*} \tilde{c}_{k-i}\right| \cos \left\{\alpha_{i}(\mathbf{x}, \tilde{\mathbf{c}})-i \tilde{\nu} T\right\}}{\frac{1}{2} \sum_{k=0}^{N_{T}-1}\left|\tilde{c}_{k}\right|^{2}}
\end{aligned}
$$

$$
\Lambda_{N_{T}}(\tilde{\mathbf{a}})=\frac{\frac{1}{2} \sum_{k=0}^{N_{T}-1}\left|x_{k}\right|^{2}\left|\tilde{c}_{k}\right|^{2}+\sum_{i=1}^{N_{T}-1}\left|\sum_{k=i}^{N_{T}-1} x_{k} \tilde{c}_{k}^{*} x_{k-i}^{*} \tilde{c}_{k-i}\right|}{\frac{1}{2} \sum_{k=0}^{N_{T}-1}\left|\tilde{c}_{k}\right|^{2}}
$$

$$
\begin{aligned}
\lambda_{n}(\tilde{\mathbf{a}})= & \frac{\frac{1}{2} \sum_{m=0}^{L-1}\left|x_{n-m}\right|^{2}\left|\tilde{c}_{n-m}\right|^{2}+\sum_{i=1}^{N-1}\left|\sum_{m=0}^{L-i-1} x_{n-m} \tilde{c}_{n-m}^{*} x_{n-m-i}^{*} \tilde{c}_{n-m-i}\right|}{\frac{1}{2} \sum_{m=0}^{L-1}\left|\tilde{c}_{n-m}\right|^{2}} \\
& -\frac{\frac{1}{2} \sum_{m=1}^{L-1}\left|x_{n-m}\right|^{2}\left|\tilde{c}_{n-m}\right|^{2}+\sum_{i=1}^{N-1}\left|\sum_{m=1}^{L-i-1} x_{n-m} \tilde{c}_{n-m}^{*} x_{n-m-i}^{*} \tilde{c}_{n-m-i}\right|}{\frac{1}{2} \sum_{m=1}^{L-1}\left|\tilde{c}_{n-m}\right|^{2}}
\end{aligned}
$$




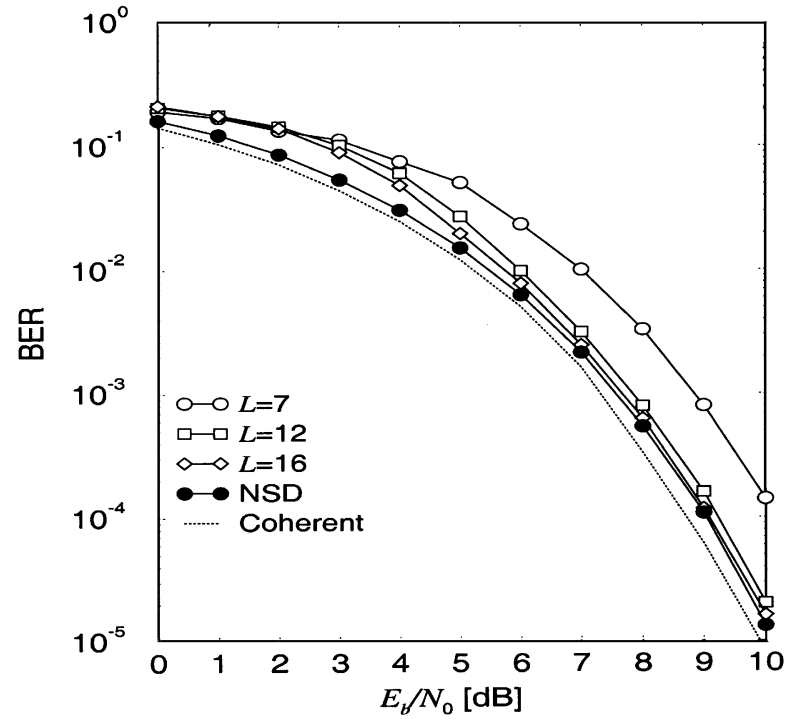

Fig. 3. BER of the proposed receiver based on (10) (white marks) for DQPSK and comparison with NSD (black marks) and coherent receivers. The frequency offset is $\nu=0$.

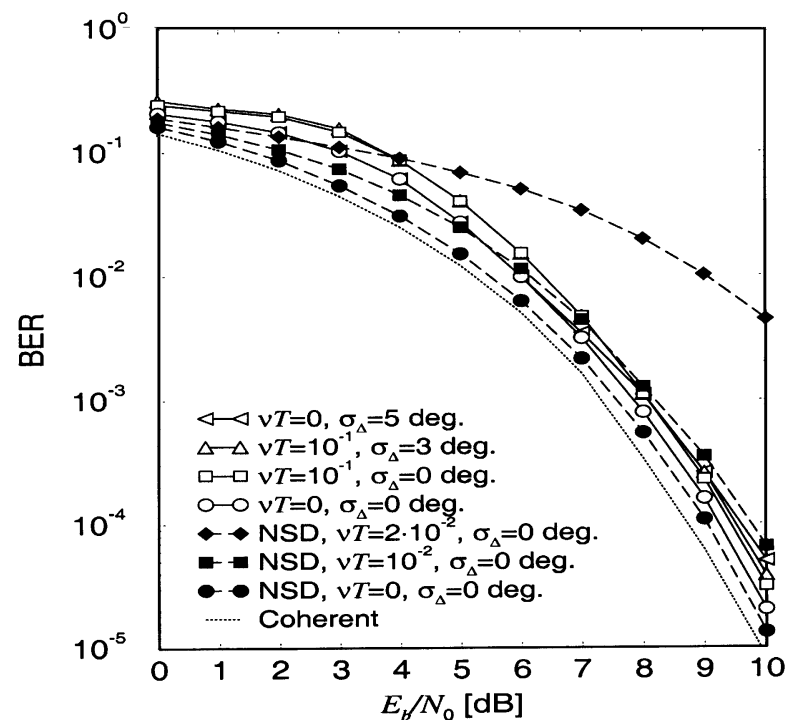

Fig. 4. BER of the proposed receiver based on (10) (white marks) for DQPSK, $N=7, L=12$, and $S=16$. The performance of an NSD receiver (black marks) with $N=7$ and $S=16$ is also shown for comparison.

may be closely approached at high signal-to-noise ratio. For $L=7$, a loss of about $1 \mathrm{~dB}$ is exhibited at a BER of $10^{-4}$. This power loss reduces to $0.2 \mathrm{~dB}$ for $L=12$ and is negligible for $L=16$.

In Fig. 4, the performance of the proposed receiver based on branch metrics (10) under dynamic channel conditions is compared with that of an NSD receiver in order to assess its robustness. The modulation format and the considered receiver are those in the previous figure with $L=12$. The transmit and receive filters have square-root raised-cosine frequency response with roll-off 0.5. ${ }^{6}$ In addition to a frequency offset, a phase noise is considered, modeled as a time-continuous Wiener

${ }^{6}$ Under dynamic channel conditions, this specification is necessary as well.

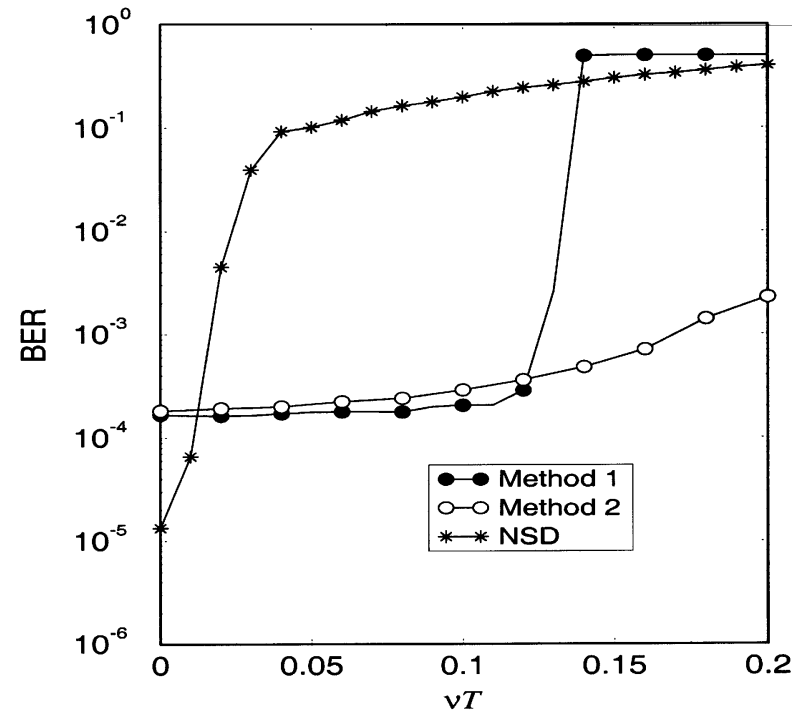

Fig. 5. BER at a signal-to-noise ratio of $10 \mathrm{~dB}$ versus the normalized frequency offset of the proposed receiver based on (10) for QPSK and $N=7, L=7$, and $S=16$. Method 1 (black marks) based on a limitation of the estimation interval and method 2 (white marks) based on DDE are considered. The performance of an NSD receiver with $N=7$ and $S=16$ is also shown for comparison.

process with incremental standard deviation over a signaling interval equal to $\sigma_{\Delta}$. The performance of the NSD receiver rapidly degrades for values of normalized frequency offset greater than $10^{-2}$, whereas the proposed receiver is practically unaffected by frequency offset up to $10^{-1}$ and phase noise up to $5^{\circ}$. As already mentioned, in the case of DQPSK, the limiting value of $|\nu T|$ is 0.125 (method 1). On the other hand, for higher values of $|\nu T|$, the receiver performance would be limited, in any case, by the front-end suboptimality and the intersymbol interference generated by the resulting mismatch. As previously noted, the algorithm used for frequency estimation is irrelevant. It has been verified that this conclusion holds for trellis-coded modulations as well, in which lower values of the signal-to-noise ratio are used.

In the previous figures, receivers based on branch metrics (10) and limitation of the estimation interval (method 1) for DQPSK were considered. Alternatively, method 2 could be used, but in this case DDE has to be employed. The different behavior of the receivers based on the two methods as well as the robustness of the proposed schemes is emphasized in Fig. 5, in which the BER versus the normalized frequency offset $\nu T$ for a signal-to-noise ratio $E_{b} / N_{0}=10 \mathrm{~dB}$ is shown for two receivers. The modulation format, the transmit and the receive filters are those of the previous figure. The curve with black marks corresponds to the performance of the proposed receiver based on branch metrics (10) with $N=7, L=7$, and $S=16$ for DQPSK with limitation of the estimation interval (method 1). The curve with white marks corresponds to a receiver based on the same branch metrics, the same values of $N, L$, and $S$, and using DDE (method 2). Frequency offset values up to $10 \%$ of the symbol rate do not affect the receiver performance. We can also note that the performance of a receiver based on method 1 rapidly degrades when the normalized frequency offset exceeds the limiting value which, for QPSK, is 0.125 . On the contrary, the receiver based on method 2 has a performance which slowly degrades with the 
TABLE I

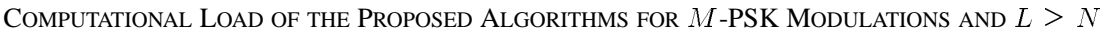

\begin{tabular}{c||c|c|c}
\hline \hline & Branch metric $(10)$ & Branch metric (16) & Branch metric (22) \\
\hline \hline Products & $2(L-1)+3 N$ & $3 L(N-2)-\frac{N}{2}(3 N-7)+1$ & $3(N-2)\left(L-\frac{N-1}{2}\right)$ \\
\hline Additions & $L+N-2$ & $L(N-2)-\frac{N}{2}(N-1)$ & $L(N-2)-\frac{N}{2}(N-5)-4$ \\
\hline ROM accesses & $L+N-1$ & - & $2(N-2)$ \\
\hline \hline
\end{tabular}

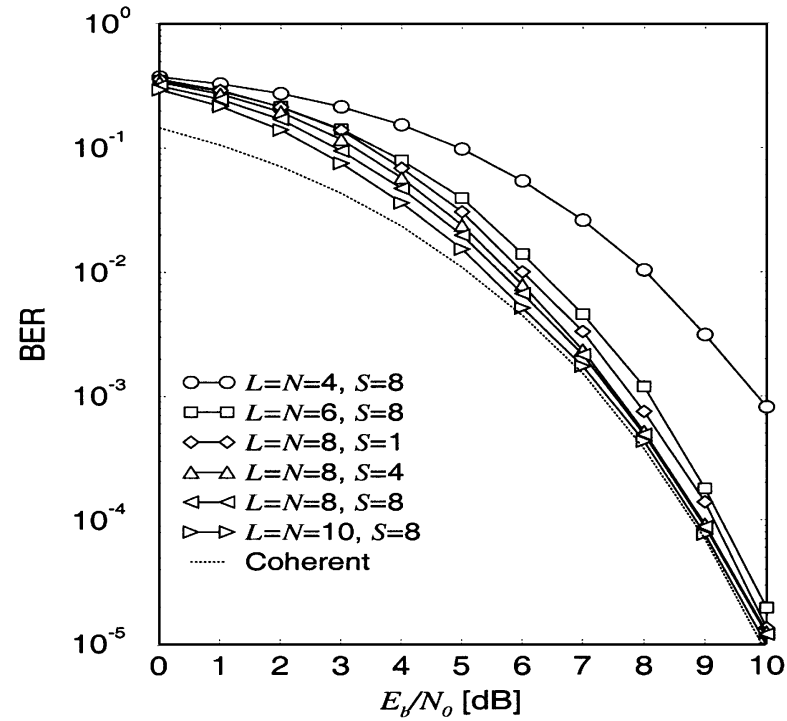

Fig. 6. BER of the proposed receiver based on (16) for $\mathrm{D}^{2} \mathrm{BPSK}$ and various values of $L, N$, and $S$. The frequency offset is $\nu=0$.

frequency offset due to the front-end suboptimality. The performance of the NSD receiver rapidly degrades already for $\nu$ equal to $1 \%$ of the signaling frequency.

The performance of the algorithm based on branch metrics (16) is shown in Fig. 6 for binary PSK with DDE (D² BPSK) and various values of $N, L$, and $S$. As in the previous case, for increasing complexity the performance rapidly approaches that of coherent detection. For high signal-to-noise ratio, a performance very close to that of a coherent detector can be attained for acceptable values of phase and frequency memory parameters.

The performance of the receiver based on (28) is shown in Fig. 7 for 16-QAM with quadrant DDE (16-D $\left.{ }^{2} \mathrm{QAM}\right)$. Curves labeled with white marks are relative to the case of absence of phase noise and frequency offset. The performance of the receiver with various values of $N, L$, and $S$, possibly in the presence of phase and frequency instabilities, is considered. In the absence of instabilities (curves with white marks), the performance approaches that of coherent detection for increasing complexity and high signal-to-noise ratio. For $N=L=14$ and $S=16$, a negligible power loss is exhibited for BER of $10^{-5}$. For these same values of $N, L$, and $S$, the performance in the presence of phase and frequency instabilities (curves with black marks) is shown, confirming the high robustness of the proposed detection schemes. In fact, it may be observed that for a normalized frequency offset of $10^{-1}$ and a phase noise standard deviation of $3^{\circ}$, the loss is only $0.6 \mathrm{~dB}$ at a BER of $10^{-5}$.

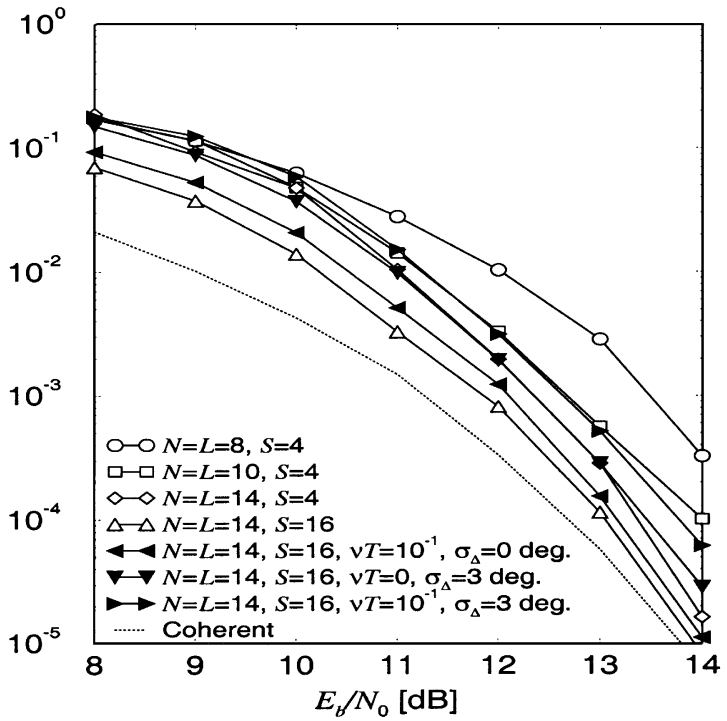

Fig. 7. BER of the proposed receiver based on (28), for $16-\mathrm{D}^{2} \mathrm{QAM}$ and various values of $L, N$, and $S$, in the absence (white marks) or in the presence (black marks) of frequency offset and phase noise.

Finally, we address the computational complexity of the proposed detection algorithms. These algorithms may be applied when a strong robustness to phase and frequency instabilities is required. These instabilities represent a problem for low bit rates. As an example, the lower the bit rate, the greater the normalized frequency offset for a given Doppler shift. Consequently, the implementation of these algorithms in low bit-rate systems is not prohibitive. In Table I, for $M$-PSK modulations, we compare the proposed algorithms with branch metrics (10), (16), or (22) in terms of number of products and additions of two complex numbers and accesses to a read-only memory (ROM), necessary to compute a single branch metric. In fact, we assume that the computation of an exponential with imaginary argument, the modulus of a complex number or its argument are performed using a ROM. For the branch metric (10), the Kay algorithm is employed to compute the frequency estimate and $L \geq N$ is assumed in all cases. We also assume that the receiver has $S$ states and RSSD is employed. As a consequence, for each state, $L-1-\log _{M} S$ symbols have to be found in the survivor history. Note that in a trellis step, when all the branch metrics are computed, some simplifications may be performed. As an example, the branch metrics departing from a given state are quite similar and, therefore, may be computed in an appropriate way to reduce the overall computational burden. In order to provide a numerical example, Table II shows the number of products, additions, and ROM accesses in the case $L=12$ and $N=7$. 
TABLE II

COMPUTATIONAL LOAD OF THE PROPOSED ALGORITHMS FOR $M$-PSK ModUlations, $L=12$, AND $N=7$

\begin{tabular}{c||c|c|c}
\hline \hline & Branch metric (10) & Branch metric (16) & Branch metric (22) \\
\hline \hline Products & 43 & 132 & 135 \\
\hline Additions & 17 & 39 & 49 \\
\hline ROM accesses & 18 & - & 10 \\
\hline \hline
\end{tabular}

\section{CONCLUSION}

Starting from the recently proposed class of NSD algorithms, this paper presents detection schemes characterized by high robustness with respect to time-varying phase and frequency instabilities. The first scheme uses PSP-based feedforward frequency estimation, whereas the other ones, based on heuristic approaches, are completely invariant to frequency offsets. Equal- and nonequal-energy signals are considered.

To resolve the inherent ambiguities of the detection schemes to phase rotations, two methods are described. The first method is based on a limitation of the allowed frequency range and estimation interval. The second method is based on the use of DDE and renders the detection strategy totally insensitive to the value of the frequency offset, except for the limitation due to the front-end suboptimality.

A performance analysis of the proposed schemes has demonstrated that strong phase noise and frequency offset are well tolerated, at the price of moderate degradations with respect to the performance exhibited by more conventional detection schemes for lower channel dynamics.

\section{APPENDIX}

An extension of the data-aided frequency estimation algorithms considered in this paper to the case of $M$-QAM signals is rather straightforward. The Rife and Boorstyn algorithm [8] is general for linear modulations, and may be directly applied to nonequal-energy signals. However, the algorithms by Kay [9] and Mengali and Morelli [10] were derived for equal-energy signals. In order to extend these algorithms to nonequal-energy signals, we can divide the observation $x_{k}$ by $c_{k}$ obtaining, under the assumption of limited values of frequency offset $\nu$

$$
y_{k} \triangleq \frac{x_{k}}{c_{k}}=e^{j(2 \pi \nu k T+\theta)}+n_{k}^{\prime}
$$

where

$$
n_{k}^{\prime} \triangleq \frac{n(k T)}{c_{k}}
$$

and $n(t) \triangleq w(t) \otimes h(-t)$. Denoting by $\sigma^{2}$ the variance of $n(k T),\left\{n_{k}^{\prime}\right\}$ are independent noise samples with zero mean and variance $\sigma^{2} /\left|c_{k}\right|^{2}$.

The algorithm by Kay is based on samples $r_{k} \triangleq \arg \left[y_{k} y_{k-1}^{*}\right]$ which, under the assumptions described in [11], may be approximated as

$$
r_{k}=\left[2 \pi \nu T+n_{I, k}^{\prime}-n_{I, k-1}^{\prime}\right]_{-\pi}^{\pi}
$$

where $n_{I, k}^{\prime}$ denotes the imaginary part of $n_{k}^{\prime}$ and $[x]_{-\pi}^{\pi}$ denotes a modulo- $2 \pi$ operation. As in the original algorithm by Kay for $M$-PSK signals, the least-square method may now be used with the only care of taking into account the fact that the noise samples $n_{I, k}^{\prime}$ have a variance which depends on the transmitted symbol $c_{k}$. As a consequence, the weighting factors of the resulting linear estimator depend on the amplitude of the transmitted sequence. Similar considerations also hold for the algorithm by Mengali and Morelli.

\section{ACKNOWLEDGMENT}

The authors wish to thank Prof. G. Picchi for several useful discussions and Ing. P. Verdi for developing part of the computer programs.

\section{REFERENCES}

[1] G. Colavolpe and R. Raheli, "Noncoherent sequence detection," IEEE Trans. Commun., vol. 47, pp. 1376-1385, Sept. 1999.

[2] — - "Noncoherent sequence detection of continuous phase modulations," IEEE Trans. Commun., vol. 47, pp. 1303-1307, Sept. 1999.

[3] D. Divsalar and M. K. Simon, "Multiple-symbol differential detection of MPSK," IEEE Trans. Commun., vol. 38, pp. 300-308, Mar. 1990.

[4] —, "Maximum-likelihood differential detection of uncoded and trellis-coded amplitude phase modulation over AWGN and fading channels-Metrics and performance," IEEE Trans. Commun., vol. 42, pp. 76-89, Jan. 1994.

[5] M. K. Simon, S. M. Hinedi, and W. C. Lindsey, Digital Communication Techniques. Englewood Cliffs, NJ: Prentice-Hall, 1995.

[6] G. Colavolpe and R. Raheli, "Theoretical analysis and performance limits of noncoherent sequence detection of coded PSK," IEEE Trans. Inform. Theory, vol. 46, pp. 1483-1494, July 2000.

[7] R. Raheli, A. Polydoros, and C. K. Tzou, "Per-survivor processing: A general approach to MLSE in uncertain environments," IEEE Trans. Commun., vol. 43, pp. 354-364, Feb./Mar./Apr. 1995.

[8] D. C. Rife and R. R. Boorstyn, "Single-tone parameter estimation from discrete-time observations," IEEE Trans. Inform. Theory, vol. 20, pp. 591-598, Sept. 1974.

[9] S. Kay, "A fast and accurate single frequency estimator," IEEE Trans. Acoust., Speech, Signal Processing, vol. 37, pp. 1987-1990, Dec. 1989.

[10] U. Mengali and M. Morelli, "Data-aided frequency estimation for burst digital transmission," IEEE Trans. Commun., vol. 45, pp. 23-25, Jan. 1997.

[11] M. Morelli and U. Mengali, "Feedforward frequency estimation for PSK: A tutorial review," Euro. Trans. Telecommun., vol. 9, pp. 103-116, Mar./Apr. 1998.

[12] H. Meyr, M. Oerder, and A. Polydoros, "On sampling rate, analog prefiltering, and sufficient statistics for digital receivers," IEEE Trans. Commun., vol. 42, pp. 3208-3214, Dec. 1994.

[13] F. Giannetti, M. Luise, and R. Reggiannini, "Simple carrier frequency rate-of-change estimators," IEEE Trans. Commun., vol. 47, pp. 1310-1314, Sept. 1999.

[14] H. L. Van Trees, Detection, Estimation and Modulation Theory. New York: Wiley, 1968, vol. I.

[15] Y. Okunev, Phase and Phase-Difference Modulation in Digital Communications. Norwood, MA: Artech House, 1997.

[16] L.-F. Wei, "Rotationally invariant convolutional channel coding with expanded signal space_Part I: $180^{\circ}$," IEEE J. Select. Areas Commun., vol. 2, pp. 659-671, Sept. 1984

[17] G. Colavolpe and R. Raheli, "Noncoherent sequence detection in frequency nonselective slowly fading channels," IEEE J. Select. Areas Commun., ser. Wireless Commun., vol. 18, pp. 2302-2311, Nov. 2000.

[18] M. V. Eyuboğlu and S. U. H. Qureshi, "Reduced-state sequence estimation with set partitioning and decision feedback," IEEE Trans. Commun., vol. 36, pp. 13-20, Jan. 1988.

[19] P. R. Chevillat and E. Eleftheriou, "Decoding of trellis-encoded signals in the presence of intersymbol interference and noise," IEEE Trans. Commun., vol. 37, pp. 669-676, July 1989. 


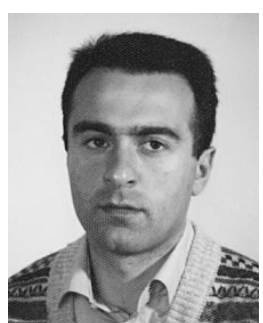

Giulio Colavolpe (S'96-A'00) was born in Cosenza, Italy, in 1969. He received the Dr.Ing. degree in telecommunications engineering (cum laude) from the University of Pisa, Pisa, Italy, in 1994, and the $\mathrm{Ph} . \mathrm{D}$. degree in information technology from the University of Parma, Parma, Italy, in 1998.

Since 1997, he has been with the University of Parma, where he is currently an Associate Professor of Telecommunications. In 2000, he was a Visiting Scientist at the Insitut Eurécom, Valbonne, France. His main research interests include digital transmission theory, channel coding, and signal processing.

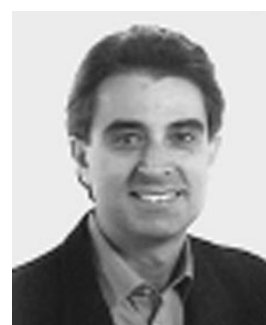

Riccardo Raheli (M'87) received the Dr.Ing. degree (Laurea) in electrical engineering (summa cum laude) from the University of Pisa, Pisa, Italy, in 1983, the M.S. degree in electrical and computer engineering from the University of Massachusetts, Amherst, in 1986, and the Ph.D. degree (Perfezionamento) in electrical engineering (summa cum laude) from the Scuola Superiore di Studi Universitari e di Perfezionamento (now "S. Anna"), Pisa, Italy, in 1987.

From 1986 to 1988, he was a Project Engineer with Siemens Telecomunicazioni, Cassina de' Pecchi (Milan), Italy. From 1988 to 1991, he was a Research Professor at the Scuola Superiore di Studi Universitari e di Perfezionamento S. Anna, Pisa, Italy. In 1990, he was a Visiting Assistant Professor at the University of Southern California, Los Angeles. Since 1991, he has been with the University of Parma, Parma, Italy, where he is currently a Professor of Telecommunications. His scientific interests are in the general area of statistical communication theory, with special attention toward digital transmission systems, data-sequence detection techniques, digital signal processing, and adaptive algorithms for telecommunications. His research activity has lead to numerous scientific publications in leading international journals and conference proceedings and a few industrial patents.

Dr. Raheli has served on the Editorial Board of the IEEE TRANSACTIONS ON COMMUNICATIONS as an Editor for Detection, Equalization, and Coding since 1999. 